\title{
A novel method to calculate the thrust of linear induction motor based on instantaneous current value
}

\author{
Jiaqing $\mathrm{MA}^{*}$, Yunpeng ZHU, Jing JIANG \\ Key Laboratory of Magnetic Levitation Technologies and Maglev Trains (Ministry of Education of China), Superconductivity and \\ New Energy R\&D Center, Southwest Jiaotong University, Chengdu 610031, China
}

\begin{abstract}
Because of the end effect, a linear induction motor (LIM) runs in an asymmetrical state even though the winding of each phase is symmetric. Based on the basic principle of the LIM, a new approach was proposed to calculate the thrust of the LIM using the instantaneous current value. A three-phase LIM model with 12 slots and a singlelayer winding was designed to validate this method. The experiments show that when the current is small, the calculated results basically agree with the experiments. The agreement becomes worse with the increase of the current because of the saturation of the primary iron core. The proposed formula is suitable when the iron core of the LIM primary is in an unsaturated state.
\end{abstract}

Key words: linear induction motor (LIM); asymmetrical state; instantaneous current value

C 2012 JMT. All rights reserved.

\section{Introduction}

$\mathrm{T}$ he operation mode of conventional rotary induction motors is to transmit the torque generated by a rotator to stressed members through the delivery mechanism. Thus the transmissions can run according to the specified mode, such as the rotation of fans and the linear motion of the vehicles. Compared with the conventional rotary induction motor, the linear induction motor (LIM) has its inherent advantages, i.e., it can run in both rotary and translational modes without any other auxiliary devices [1-4].

Yamamamura [1] reviewed the basic theory and analyzed the structures of several types of LIMs in details. He stated that the essential difference between the LIM and the rotary induction motor is the open linear air gap due to the entry and exit end of the LIM. He also presented the three-dimensional analysis of the LIM to analyze its special end effects. Long [2] presented a universal approach for the LIM's magnetic design. He developed a set of formulas to calculate the main parameters of LIM such as the pitch, the numbers of pole pairs, the apparent power etc. Ye [3] deduced a formula from Maxwell equations to calculate the thrust of unit wave length of the LIM.

The end effects of the LIM include the longitudinal and transverse components [5]. Zhang [6] analyzed the

Received May 10, 2012; revision accepted Jun. 12, 2012

${ }^{*}$ Corresponding author. E-mail: majiaqin_9230@qq.com (J.Q. MA)

(C) 2012 JMT. All rights reserved

doi: 10.3969/j.issn.2095-087X.2012.02.003 static longitudinal end effects of the LIM, and presented the formulas to calculate the air gap magnetic field pulsating coefficient, the magnetic flux density increasing coefficient, and the longitudinal equivalent magnetic leakage length according to electromagnetic field theory. Bolton [7] discussed the transverse effects of the LIM with sheet-rotor. He considered that the flux redistribution due to the transverse effect could influence the performance considerably for certain motors. Long [8] proposed a general deriving method for the LIM's equivalent circuit from the complex power equivalent relationship in the electric circuit and magnetic field analysis with consideration of end effect.

In summary, substantial efforts [9-15] have been investigated in the theoretical study of LIM. In order to analyze the operation characteristics of an LIM, a general approach is commonly used to describe the current in primary winding as a continuous current sheet. This current sheet that changes continuously both in the temporal and spatial domain is called the traveling wave current layer. This general method, however, is not easy for calculation.

In this paper, the instantaneous current value of each primary slot is used to calculate the thrust of LIM. To do this, the instantaneous current in each slot has to be calculated first, and the thrust generated by each slot is determined. All thrusts finally need to be summed up to form the final formula. We designed a three-phase LIM model with 12 slots and a single-layer winding to validate this method. 


\section{The formation of traveling current}

An LIM model is shown in Fig. 1, where $2 a, b$ and $c$ represent the width, height, and length of the primary, respectively, and $2 l, m$ and $n$ the width, height, and length of the secondary, respectively; $b_{0}$ is the distance between the first slot and the left edge of the LIM; $b_{k}$ is the pitch of the primary teeth; $\delta$ is the distance of the primary and secondary; and $\delta_{\mathrm{c}}$ is the distance of the top side between the primary and the winding centers.

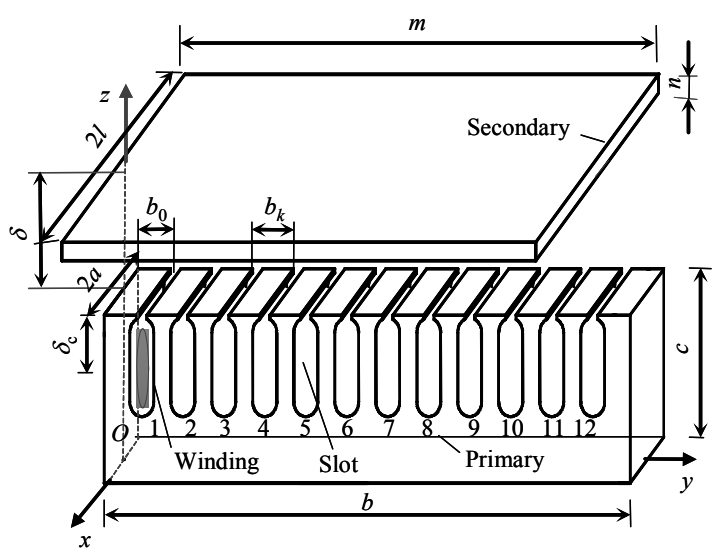

Fig. 1 An LIM model

For the convenience of analysis, we assume:

(1) The primary and the secondary are parallel, and the centerlines of their $x-y$ planes are aligned.

(2) The relative motion between the primary and the secondary takes place only in the $y$ direction, and the primary is static.

(3) The positive direction of the current is on the negative direction of the $x$ axis.

Fig. 2 shows the mode of the windings embedded in the secondary.

When windings $A, B$, and $C$ are in a balanced state, the amplitudes of windings are equal and their phase difference is $120^{\circ}$. Normalized operating current is shown in Fig. 3. To illustrate the formation of the discrete trav-

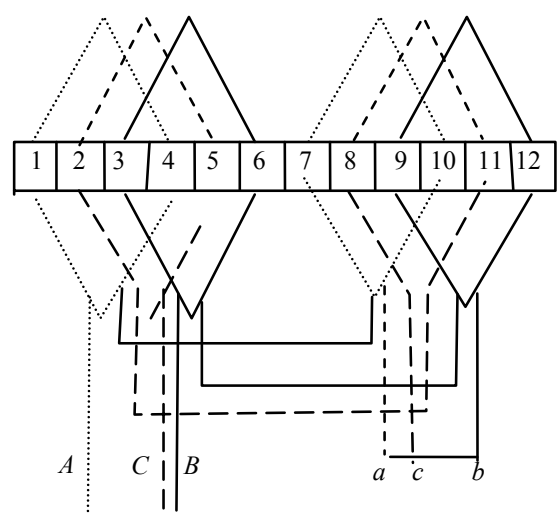

eling wave current in $x$ direction in the primary of the LIM, two arbitrary points $t_{1}$ and $t_{2}$ are selected within any half of period.

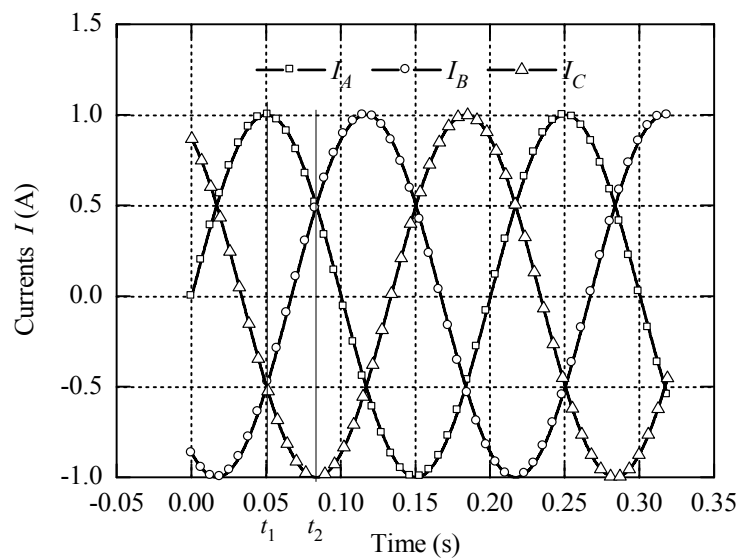

Fig. 3 Three phase currents (normalized)

At $t_{1}$ :

$I_{A}=-2 I_{B}=-2 I_{C}=1 \mathrm{~A}$.

At $t_{2}$ :

$I_{A}=I_{B}=-\frac{1}{2} I_{C}=\frac{1}{2} \mathrm{~A}$.

The instantaneous values of each slot current are calculated by Eqs. (1) and (2), and listed in Table 1. The flowing current on 12 slots illustrates the formation of the traveling wave current as shown in Fig. 4. The motion direction of the secondary is consistent with that of the traveling wave current. If any two phases are changed, the direction of the wave velocity turns opposite.

Table 1 Instantaneous current value in each slot A

\begin{tabular}{ccccc}
\hline $\begin{array}{c}\text { Slot } \\
\text { No. }\end{array}$ & Wire & $t_{1}$ & $t_{2}(A B C)$ & $t_{2}(A C B)$ \\
\hline 1 & $+\mathrm{A}$ & 1 & $1 \backslash 2$ & $1 \backslash 2$ \\
2 & $-\mathrm{C}$ & $1 \backslash 2$ & 1 & $-1 \backslash 2$ \\
3 & $+\mathrm{B}$ & $-1 \backslash 2$ & $1 \backslash 2$ & -1 \\
4 & $-\mathrm{A}$ & -1 & $-1 \backslash 2$ & $-1 \backslash 2$ \\
5 & $+\mathrm{C}$ & $-1 \backslash 2$ & -1 & $1 \backslash 2$ \\
6 & $-\mathrm{B}$ & $1 \backslash 2$ & $-1 \backslash 2$ & 1 \\
7 & $+\mathrm{A}$ & 1 & $1 \backslash 2$ & $1 \backslash 2$ \\
8 & $-\mathrm{C}$ & $1 \backslash 2$ & 1 & $-1 \backslash 2$ \\
9 & $+\mathrm{B}$ & $-1 \backslash 2$ & $1 \backslash 2$ & -1 \\
10 & $-\mathrm{A}$ & -1 & $-1 \backslash 2$ & $-1 \backslash 2$ \\
11 & $+\mathrm{C}$ & $-1 \backslash 2$ & -1 & $1 \backslash 2$ \\
12 & $-\mathrm{B}$ & $1 \backslash 2$ & $-1 \backslash 2$ & 1 \\
\hline
\end{tabular}

Fig. 2 Three-phase single-layer winding in LIM 
In a balanced state, the value of the traveling current can be expressed as:

$$
\begin{aligned}
& I_{(t, k)}=\sqrt{2} N I_{m A} \sin \left[\left(-\omega t+\theta_{A 0}\right)+(2 k+1) \times \frac{2 b_{n} \pi}{2 p \tau}\right] \\
& =\sqrt{2} N I_{m A} \sin \left[\left(-\omega t+\theta_{A 0}\right)+(2 k+1) \times \frac{b_{n} \pi}{p \tau}\right], \\
& k=1,2,3, \ldots, 12,
\end{aligned}
$$

where $k$ is the serial number of the slots; $I_{m A}$ is RMS current of phase $A ; p$ denotes pole pairs of the primary ( 2 in this paper); $\theta_{A 0}$ is initial phase angle of phase $A$ ( 0 in this paper); $b_{n}$ is slot pitch; $\tau$ is pole pitch (3 times $b_{n}$ in this paper), and $N$ is the turns of each winding.

If phases $A$ and $B$ are exchanged, then

$$
\begin{aligned}
I_{(t, k)} & =\sqrt{2} N I_{m A} \sin \left[\left(\omega t+\theta_{A 0}\right)+(2 k+1) \times \frac{b_{n} \pi}{p \tau}\right], \\
k & =1,2,3, \cdots, 12 .
\end{aligned}
$$

In the case of asymmetrical factory, Eq. (3) is rewritten as

$$
\begin{aligned}
I_{(t, k)} & =\sqrt{2} N I_{m k} \sin \left[\left(-\omega t+\theta_{A 0}+\varphi_{k}\right)+(2 k+1) \times \frac{b_{n} \pi}{p \tau}\right], \\
k & =1,2,3, \cdots, 12,
\end{aligned}
$$

when $k=1,4,7,10, I_{m k}$ is equal to the RMS of phase $A$; $\varphi_{k}$ is $\varphi_{A}$ when $k=2,5,8,11 ; I_{m k}$ is equal to the RMS of phase $B$; and $\varphi_{k}$ is $\varphi_{B}$ when $k=3,6,9,12 ; I_{m k}$ is equal to the RMS of phase $C$ and $\varphi_{k}$ is $\varphi_{C}$. When $I_{m k}=I_{m A}$, and $\varphi_{A}=\varphi_{B}=\varphi_{C}$, Eq. (5) is exactly equal to Eq. (3).

The derivation of the wave velocity can be obtained from Fig. 4. Then, one has

$$
v_{s}=f \lambda=\frac{1}{T} 2 \tau=2 f \tau \text {. }
$$

where $v_{s}$ is the velocity of the traveling current layer; $f$ is the frequency of power supply; $T$ is the period of power supply; and $\tau$ is pole pitch.

We can see in Fig. 4 that if the currents of $B$ and $C$ are exchanged, the velocity direction of the traveling current layer turns to opposite. Furthermore, if any two phases of $A, B$, and $C$ are exchanged arbitrarily, the direction is inevitably opposite.

\section{The formation of traveling wave in the magnetic field}

A straight wire carrying current induces magnetic field around it [9-10]. If a number of wires, each of which has a suitable variable phase angle and amplitude of current, are arranged in a reasonable order, the traveling magnetic field is produced. In an LIM, when the rotator with closed wires is static, there is a relative motion between the traveling field and the rotator. Then the induction current flows and exerts force on the straight wire. This is the general principle of the induction motors.

The magnetic field is generated by the flow of the current. If other conditions are constant, the strength of the magnetic field is related to the amplitude of the current. The velocity of traveling wave magnetic field is consistent with that of the traveling wave current as shown in Fig. 5.

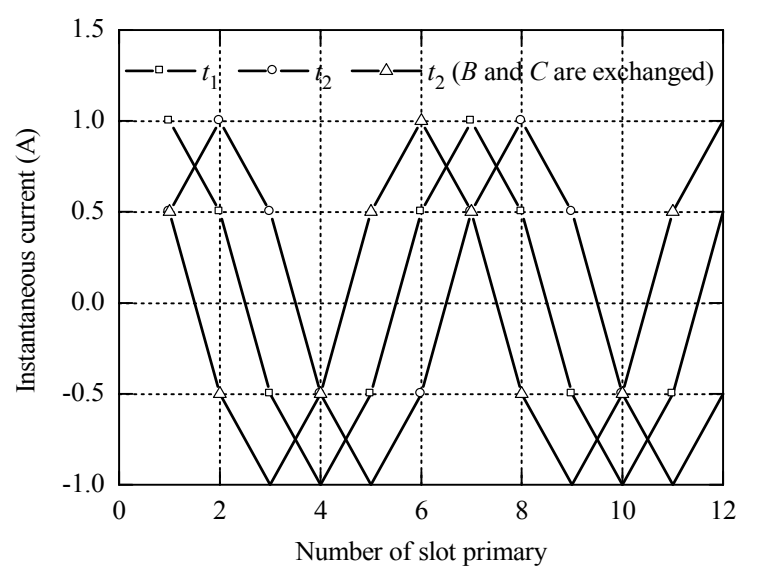

Fig. 4 The formation of the traveling wave current

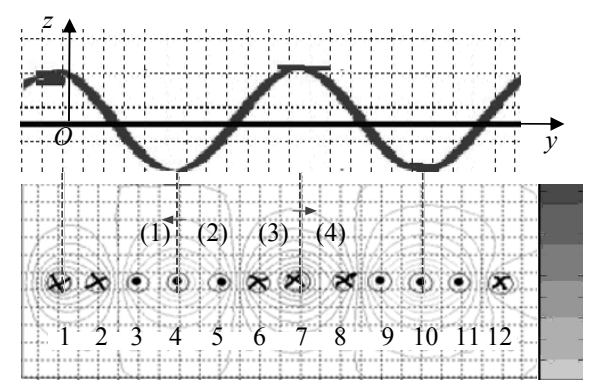

Fig. 5 Formation of traveling wave in the magnetic field

Fig.5 shows the magnetic field generated by the flowing current of primary slots at time $t_{1}$ shown in Fig. 3 .

It should be emphasized that the magnetic field of a traveling wave is continuous both in the temporal and spatial domain. The velocity of traveling magnetic field is stated as

$$
\boldsymbol{v}_{s m}=\boldsymbol{v}_{s} \text {. }
$$

In contrast to the magnetic field of the traveling wave, the secondary with an electric conductor sheet has a motion, and its velocity is given by

$$
\boldsymbol{v}_{r}=\left(v_{r x} \boldsymbol{e}_{x}+v_{r y} \boldsymbol{e}_{y}+v_{r z} \boldsymbol{e}_{z}\right)=-\left(\boldsymbol{v}_{s m}-\boldsymbol{v}\right),
$$

where $\boldsymbol{v}_{r}$ is the relative velocity of the secondary when taking traveling wave magnetic field as reference; $v$ is the ab- 
solute velocity of the secondary when taking the primary as reference; $\boldsymbol{e}_{x}, \boldsymbol{e}_{y}$, and $\boldsymbol{e}_{z}$ are unit vectors of orthogonal coordinate system, respectively; and $v_{r x}, v_{r y}$, and $v_{r z}$ are the corresponding components of each axis respectively.

Because the motion only occurs in $y$ direction, $v_{r x}$ and $v_{r z}$ are equal to zero. Thus, the slip is given as

$$
s=\frac{\boldsymbol{v}_{s m y}-\boldsymbol{v}_{y}}{\boldsymbol{v}_{s m y}}
$$

where $\boldsymbol{v}_{s m y}$ is the component of $\boldsymbol{v}_{s m}$ in the $y$ direction.

Then

$$
\boldsymbol{v}_{r y}=-\left(\boldsymbol{v}_{s m y}-\boldsymbol{v}_{y}\right)=-s \boldsymbol{v}_{s m y} .
$$

To calculate the magnetic field on each slot, polar coordinate is used at first and then replaced with the orthogonal coordinate. According to the magnetic disribution as shown in Fig. 6, the magnetic field is given by

$$
\boldsymbol{B}(r, \phi)=\frac{\mu_{0} I}{2 \pi r} \boldsymbol{e}_{\phi}
$$

where $\boldsymbol{B}(r, \phi)$ is magnetic flux density in polar coordinate; $\mu_{0}$ is air permeability; $I$ is the current of straight wire; $r$ is the distance of the wire and some point around the wire; and $\boldsymbol{e}_{\phi}$ is the unit vector in $\phi$ direction in polar coordinate.

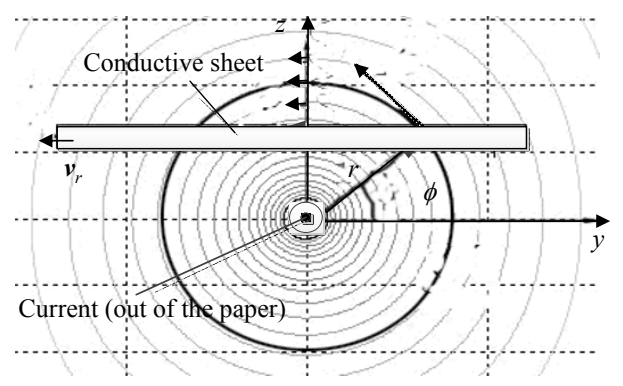

Fig. 6 Distribution of the magnetic field around the straight wire with current (the direction of the current is out of the paper)

The relationship of the polar and the orthogonal coordinate is

$$
\boldsymbol{e}_{\phi}=-\boldsymbol{e}_{y} \sin \phi+\boldsymbol{e}_{z} \cos \phi .
$$

Thus,

$$
\begin{gathered}
\boldsymbol{B}(x, y, z)=\left(B_{x} \boldsymbol{e}_{x}+B_{y} \boldsymbol{e}_{y}+B_{z} \boldsymbol{e}_{z}\right)= \\
\frac{\mu_{0} K_{\mu} I_{(t, k)}}{2 \pi\left(y^{2}+z^{2}\right)}\left(-z \boldsymbol{e}_{y}+y \boldsymbol{e}_{z}\right)= \\
\frac{\mu_{0} \mu_{r} I_{(t, k)}}{2 \pi r}\left(-\boldsymbol{e}_{y} \sin \phi+\boldsymbol{e}_{z} \cos \phi\right) .
\end{gathered}
$$

\section{The process of calculating thrust}

Fig. 7 shows the force at 4 points in the magnetic field (marked (1), (2), (3), and (4) in Fig. 5).

As shown in Fig. 7, we take the 4th slot as an instance to formulate the thrust. The inductive electromotive force is generated at a very small portion on the secondary conductive sheet. The area of this portion is $\mathrm{d} y \mathrm{~d} z$ and the length is $2 l$. Thus the inductive electromotive force is described as:

$$
\varepsilon_{i n}=2 \int_{l} \boldsymbol{v}_{r} \times \boldsymbol{B} \cdot \mathrm{d} \boldsymbol{l}=-2 v_{r y} B_{z} l,
$$

where $\mathrm{d} \boldsymbol{l}=-l \cdot \boldsymbol{e}_{x}$, and $B_{z}$ is magnetic flux density in the $z$ direction. Thus the inductive currency is

$$
\mathrm{d} I_{\text {in }}=\frac{\mathrm{d} \varepsilon_{\text {in }}}{R+R_{\text {end }}}=\varepsilon_{\text {in }} / \rho \frac{2\left(l+l_{\text {end }}\right)}{\mathrm{d} s}=-\frac{l v_{r y} B_{z} \mathrm{~d} y \mathrm{~d} z}{\rho\left(l+l_{\text {end }}\right)},
$$

where $\rho$ is the resistivity of the secondary material, and $l_{\text {end }}=0$.

Hence the force generated by this small participation is

$$
\begin{aligned}
& \mathrm{d} \boldsymbol{f}=\left(\mathrm{d} f_{x} \boldsymbol{e}_{x}+\mathrm{d} f_{y} \boldsymbol{e}_{y}+\mathrm{d} f_{z} \boldsymbol{e}_{z}\right)=2 \mathrm{~d} I_{\text {in }} \mathrm{d} \boldsymbol{l} \times \boldsymbol{B}= \\
& 2 l \mathrm{~d} I_{\text {in }}\left(B_{z} \boldsymbol{e}_{y}-B_{y} \boldsymbol{e}_{z}\right) .
\end{aligned}
$$

In (16), the force in the $z$ direction is the normal force and that in the $y$ direction is the thrust.

Then the force $\mathrm{d} f$ is obtained by integrating Eqs. (13), (15) and (16):

$$
\begin{aligned}
& \mathrm{d} \boldsymbol{f}=2 l \mathrm{~d} I_{\text {in }}\left(B_{z} \boldsymbol{e}_{y}-B_{y} \boldsymbol{e}_{z}\right)=-\frac{l^{2} \mu_{0}{ }^{2} K_{\mu}{ }^{2} I^{2}{ }_{(t, k)} v_{r y}}{2 \pi^{2} \rho} \times \\
& \left(\frac{z^{2}}{\left(y^{2}+z^{2}\right)^{2}} \boldsymbol{e}_{y}+\frac{z y}{\left(y^{2}+z^{2}\right)^{2}} \boldsymbol{e}_{z}\right)= \\
& -\frac{2 l^{2} v_{r y}}{\rho\left(l+l_{\text {end }}\right)}\left(B_{z}^{2} \boldsymbol{e}_{y}-B_{z} B_{y} \boldsymbol{e}_{z}\right) \mathrm{d} y \mathrm{~d} z .
\end{aligned}
$$

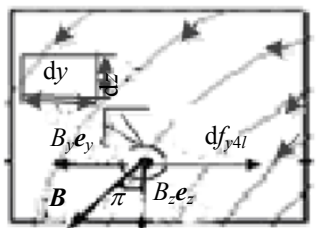

(a) Left of the 4th stot

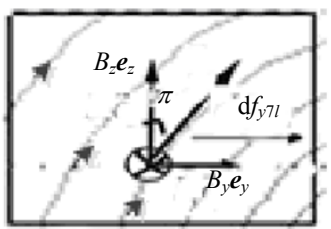

(c) Left of the 7th slot

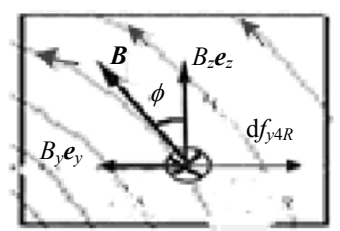

(b) Right of the 4th slot

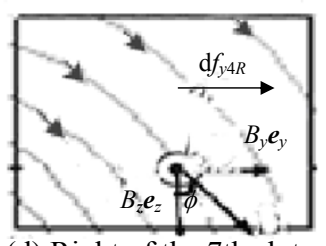

(d) Right of the 7th slot
Fig. 7 The force generated by each slot embedded with wire 
Fig. 7(a) indicates the force on the left part of the 4th slot of the secondary sheet. This force is generated by the moving magnetic field, and this field is caused by the instantaneous current flowing on the 4th slot. The forces caused by other slots as shown in Fig. 5 are similar to this case.

Then the force generated by the $k$ th slot is

$$
\begin{aligned}
& \boldsymbol{f}_{(k, t)}=\iint_{y z} \frac{l^{2} \mu_{0}{ }^{2} K_{\mu}{ }^{2} I^{2}{ }_{(t, k)} v_{r y}}{2 \pi^{2} \rho} \times \\
& \left(\frac{z^{2}}{\left(y^{2}+z^{2}\right)^{2}} \boldsymbol{e}_{y}+\frac{z y}{\left(y^{2}+z^{2}\right)^{2}} \boldsymbol{e}_{z}\right) \mathrm{d} y \mathrm{~d} z .
\end{aligned}
$$

The total force is

$$
\begin{aligned}
\boldsymbol{F}(t) & =-\sum_{k=1}^{12} \boldsymbol{f}_{(k, t)}=-\frac{l^{2} \mu_{0}{ }^{2} K_{\mu}{ }^{2} v_{r y}}{2 \pi^{2} \rho} \times \\
& \sum_{k=1}^{12}\left[I^{2}{ }_{(t, k)} \iint_{y}\left(\frac{z^{2}}{\left(y^{2}+z^{2}\right)^{2}} \boldsymbol{e}_{y}+\frac{z y}{\left(y^{2}+z^{2}\right)^{2}} \boldsymbol{e}_{z}\right) \mathrm{d} y \mathrm{~d} z\right]= \\
& -\frac{l^{2} \mu_{0}{ }^{2} K_{\mu}{ }^{2} v_{r y} \boldsymbol{e}_{y}}{2 \pi^{2} \rho} \times \\
& \sum_{k=1}^{12}\left(I^{2}{ }_{(t, k)} \int_{\delta_{c}+\delta}^{\delta_{c}+\delta+n} \int_{y_{0}}^{y_{0+m}} \frac{z^{2}}{\left(y^{2}+z^{2}\right)^{2}} \mathrm{~d} y \mathrm{~d} z\right) .
\end{aligned}
$$

Since the motion occurs only in $y$ direction as shown in Fig. 1, even when all slots are considered, the position of the force is changed only in $y$ direction. Hence equation (19) is modified as

$$
\boldsymbol{F}(t)=-\frac{l^{2} \mu_{0}^{2} K_{\mu}^{2} v_{r y} \boldsymbol{e}_{y}}{2 \pi^{2} \rho} \sum_{k=1}^{12}\left(I_{(t, k)}^{2} \int_{\delta_{c}+\delta}^{\delta_{c}+\delta+n} \int_{y_{0}}^{y_{0+m}} \frac{z^{2}}{\left(y-\left[b_{0}+(x-1) b_{n}\right]^{2}+z^{2}\right)^{2}} \mathrm{~d} y \mathrm{~d} z\right)
$$

If the slip is taken into consideration, Eq. (20) is rewritten as:

$$
\begin{aligned}
& \boldsymbol{F}(t)=\frac{l^{2} \mu_{0}^{2} K_{\mu}^{2} s v_{s m y} \boldsymbol{e}_{y}}{2 \pi^{2} \rho} \times \\
& \sum_{k=1}^{12}\left(I_{(t, k)}^{2} \int_{\delta_{\mathrm{c}}+\delta}^{\delta_{\mathrm{c}}+\delta+n} \int_{y_{0}-\left[b_{0}+(x-1) b_{n}\right]}^{y_{0+m}-\left[b_{0}+(x-1) b_{n}\right]} \frac{z^{2}}{\left(y^{2}+z^{2}\right)^{2}} \mathrm{~d} y \mathrm{~d} z\right) .
\end{aligned}
$$

From Eq. (21), the relationships between the thrust and the current, the thrust and the slip, as well as the thrust and the gap can easily be obtained.

\section{Comparison of calculated results and measured data}

To validate Eq. (21), we observed the operation of a three-phase LIM with the single-layer winding as shown in Fig. 8. The maglev train is levitated above the permanent magnetic guideway with a height of $10 \mathrm{~mm}$ and can only move in one direction because of the characters of the high temperature superconducting materials. The secondary of the LIM is pasted on the train, and the primary and the force meter are fixed on the ground. When the LIM is in operation, the forcemeter shows the value of the thrust. The PF3400 is an instrument to measure the three-phase parameters of LIM such as currents, voltages, power factors, etc. We can get the different thrusts by changing the winding currents which are measured by PF3400.

Even though the impedance of each of the winding is balanced, the running states of phases $A, B$, and $C$ are not completely symmetrical due to the end effect of LIM. We assume that the phases $A, B$ and $C$ are symmetrical and in Eq. (21) $s=1$.
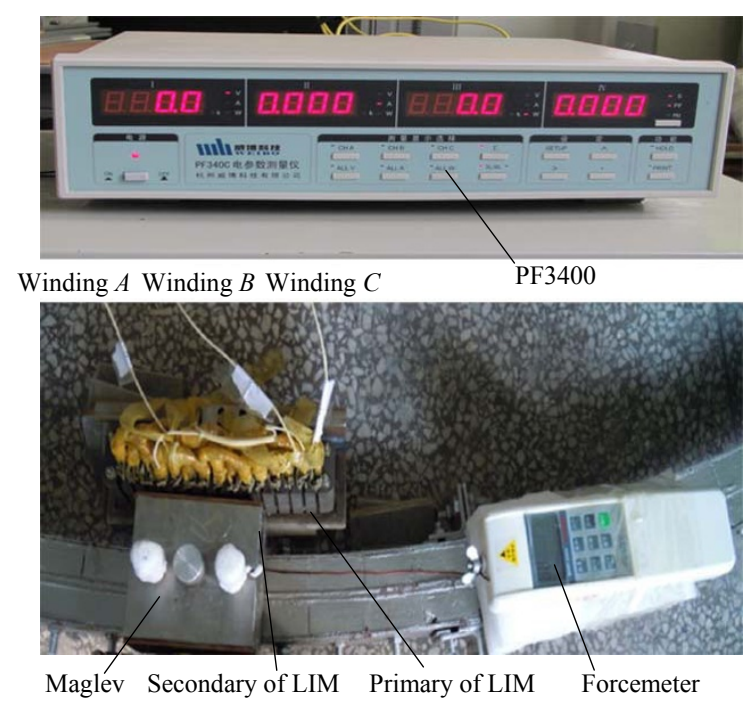

Fig. 8 The measurement of the thrust and currents

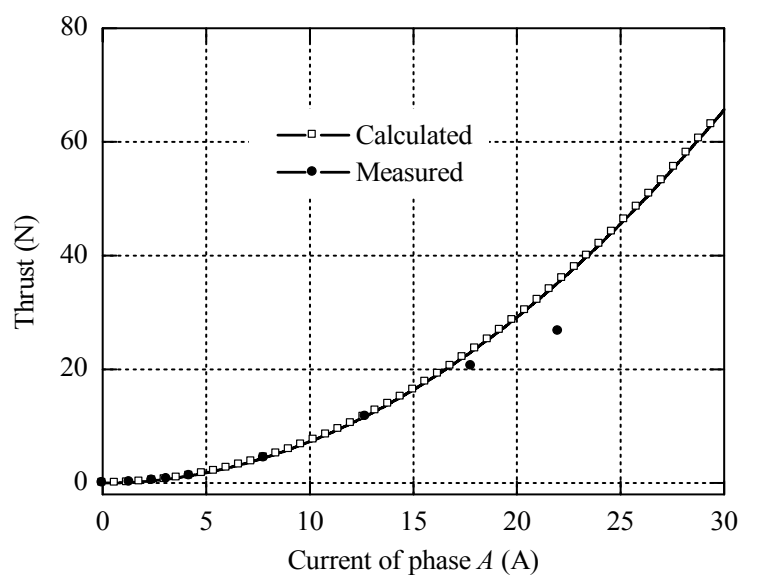

Fig. 9 Comparison of two results 
The current of phase $A$ is used to calculate the thrust and the calculated results are compared with the measured results shown on the forcemeter. As shown in Fig. 9, the current for the calculated results ranges from 0 to $30 \mathrm{~A}$, and the measured data corresponds to the currents at 3.6, 4.9, 6.0, 6.8, 7.8, 11.4, 16.3, and 21.5 A, respectively. When the current is small, two results basically agree with each other. The agreement degraded with the increase of the current because of the saturation of the primary iron core. Eq. (21) is suitable when the iron core of the LIM primary is in an unsaturated state.

Because the thrust is continuous in the time domain, the secondary of the LIM is forced at any time point. The calculated thrust with the slip of 1 and current of $20 \mathrm{~A}$ is shown in Fig. 10. We can see that the fluctuation is so small that the thrust variation can be ignored.

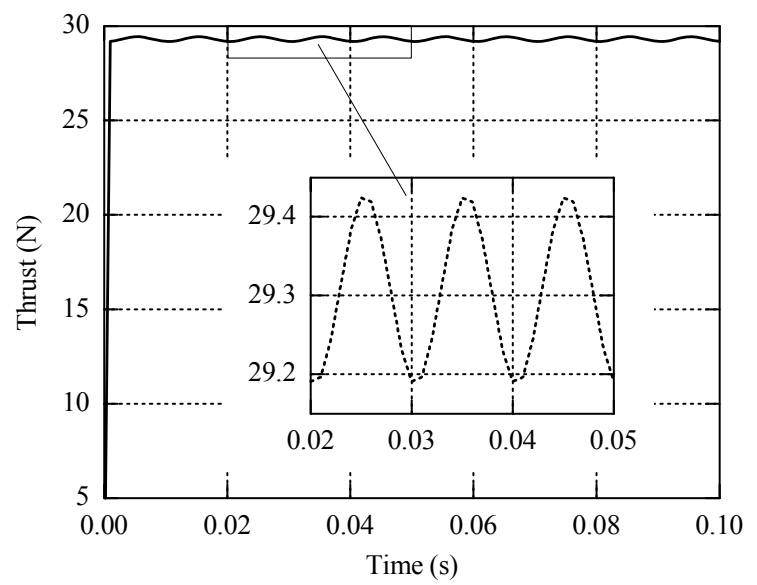

Fig. 10 The relationship between the thrust and the time

\section{Conclusions}

According to the LIM's principle, when the motor is in the running state, the traveling wave magnetic field is formed in the gap between the primary and the secondary. According to this principle, the instantaneous current value is used to deduce the thrust of the LIM with copper secondary. The calculated results agree with the experimental data, demonstrating that this approach is feasible and applicable. The calculation formula clearly shows the relationships between the thrust and the current, the thrust and the slip, as well as the thrust and the air gap, etc.

\section{Acknowledgements}

This work was supported by the National Natural Science Foundation of China (Nos. 50588201, 50672078, and 50872116), the National Basic Research Program (973 program, No. 2007CB616906), the Australian Research Council (Grant No. DP0559872 and DP0881739), and the PCSIRT of the Ministry of Education of China (No. IRT0751).

\section{References}

[1] S. Yamamamura, Theory of Linear Induction Motor, Tokyo: University of Tokyo Press, 1978.

[2] X.L. Long, Theory and Magnetic Design Method of Linear Induction Motor, Beijing: China Science Publishing, 2006 (in Chinese).

[3] Y.Y. Ye, The Principle and Application of Linear Machine, Beijing: China Machine Press, 2000 (in Chinese).

[4] A.I. Bolde, S.A. Nasar, Linear Motion Electromagnetic systems, New York: John Wiley and Sons Inc. Press, 1985.

[5] E.R. Laithwaite, S.A. Nasar, Linear motion electrical machines, In: Proc. of the IEEE, 1970, 58(4): 537-541.

[6] G.Y. Zhang, Research on the static longitudinal end effect of linear electric machine, Transactions of China Electrotechnical Society, 1999, 14(5): 18-21 (in Chinese).

[7] H. Bolton, Transverse edge effect in sheet-rotor induction motors, PIEE, 1969, 116(5): 725-731.

[8] X.L. Long, A general method deriving the equivalent circuit of linear induction motor, Transactions of China Electrotechnical Society, 1993, 8(4): 55-60 (in Chinese).

[9] J.D. Kraus, Electromagnetics, New York: McGraw-Hill, 1984.

[10] E.M. Purcell, Electricity and Magnetism, New York: McGraw-Hill, 1985.

[11] W. Xu, J.G. Zhu, Y.C. Zhang, et al., Equivalent circuits for single-sided linear induction motors, IEEE Transactions on Industry Applications, 2010, 46(6): 2410-2413.

[12] C.S. Chen, Equation for Mathematical Physics, Nanjing: Southeast University Press, 2002 (in Chinese).

[13] R. Hellinger, P. Mnich, Linear motor-powered transportation: history, present status, and future outlook, Proc. IEEE, 2009, 97(11): 1892-1900.

[14] S.J. Chapman, Electric Machinery Fundamentals (4th Edition), New York: McGraw-Hill, 2005.

[15] Y.Q. Tang, Electromagnetic Field of Electric Machine, Beijing: Science Press, 1998 (in Chinese).

(Editor: Yao ZHOU) 